\title{
Organization of the mycobacterial cell wall: a nanoscale view
}

\author{
David Alsteens • Claire Verbelen • Etienne Dague • \\ Dominique Raze • Alain R. Baulard • Yves F. Dufrêne
}

Received: 25 September 2007 / Accepted: 30 October 2007 / Published online: 28 November 2007

(C) Springer-Verlag 2007

\begin{abstract}
The biosynthesis of the Mycobacterium tuberculosis cell wall is targeted by some of the most powerful antituberculous drugs. To date, the molecular mechanisms by which these antibiotics affect the cell wall characteristics are not well understood. Here, we used atomic force microscopy - in three different modes - to probe the nanoscale surface properties of live mycobacteria and their modifications upon incubation with four antimycobacterial drugs: isoniazid, ethionamide, ethambutol, and streptomycine. Topographic imaging, combined with quantitative surface roughness analysis, demonstrated that all drugs induce a substantial increase of surface roughness to an extent that correlates with the localization of the target (i.e., synthesis of mycolic acids, arabinogalactans, or proteins). Chemical force microscopy with hydrophobic tips revealed that the structural alterations induced by isoniazid and ethambutol were correlated with a dramatic decrease of cell surface hydrophobicity, reflecting the removal of the outermost mycolic acid layer. Consistent with this finding, tapping mode imaging, combined with immunogold labeling, showed that the two drugs lead to the massive exposure of hydrophilic lipoarabinomannans at the surface. Taken together, these structural, chemical, and immunological data provide novel insight into the action mode of antimyco-
\end{abstract}

D. Alsteens $\cdot$ C. Verbelen $\cdot$ E. Dague $\cdot$ Y. F. Dufrêne $(\bowtie)$

Unité de Chimie des Interfaces,

Université Catholique de Louvain,

Croix du Sud 2/18,

1348 Louvain-la-Neuve, Belgium

e-mail: dufrene@cifa.ucl.ac.be

D. Raze $\cdot$ A. R. Baulard

INSERM U629, Mécanismes Moléculaires de la Pathogénie

Bactérienne, Institut Pasteur de Lille,

IFR142, 1 rue du Professeur Calmette,

59019 Lille Cedex, France bacterial drugs, as well as into the spatial organization of the mycobacterial cell wall.

Keywords Antimycobacterial drugs · Cell walls . Chemical force microscopy Immunogold labeling . Hydrophobicity · Live cell imaging

\section{Introduction}

The Gram-positive bacterial pathogen Mycobacterium tuberculosis infects over one-third of the population in the world, causing annually nine million new cases and two million deaths [19]. The highly complex mycobacterial cell wall envelope plays essential roles in controlling the growth of the bacteria, its survival in the infected host, and the immunologic response $[9,12,15]$. Our present day understanding of its structure reveals a three-compartment entity composed of a plasma membrane; the cell wall core; and the extractable noncovalently linked glycans, lipids, and proteins (Fig. 1). The insoluble cell wall core is an insoluble matrix of crosslinked peptidoglycans linked to arabinogalactans, esterified at the distal ends to mycolic acids [3]. Although these major cell wall constituents are relatively well described, their three-dimensional distribution remains poorly understood and somewhat controversial $[10,16]$. In particular, while a large proportion of lipoarabinomannan (LAM) and mannosylated phosphatidyl inositol (LM and PIMs) are proposed to be anchored into the cell membrane via their lipidic portion, they are also known to be present in the outermost portion of the envelope, which allows them to interact with the immune system $[23,26]$. Thus, it is still unclear whether hydrophobic mycolic acids are present in the form of a continuous, outermost layer on the surface or whether hydrophilic 
Fig. 1 Schematic representation of the mycobacterial cell wall architecture. The three main constituents are mycolylarabinogalactan, LAM, and mycolic acids. It is unclear whether hydrophobic mycolic acids are present in the form of a continuous, outermost layer on the surface or whether hydrophilic polysaccharides, particularly LAMs, are embedded in this layer. Arrows indicate the action sites of the four antimycobacterial drugs investigated here: isoniazid $(I N H)$, ethionamide $(E T H)$, ethambutol $(E M B)$, and streptomycin $(S T R)$

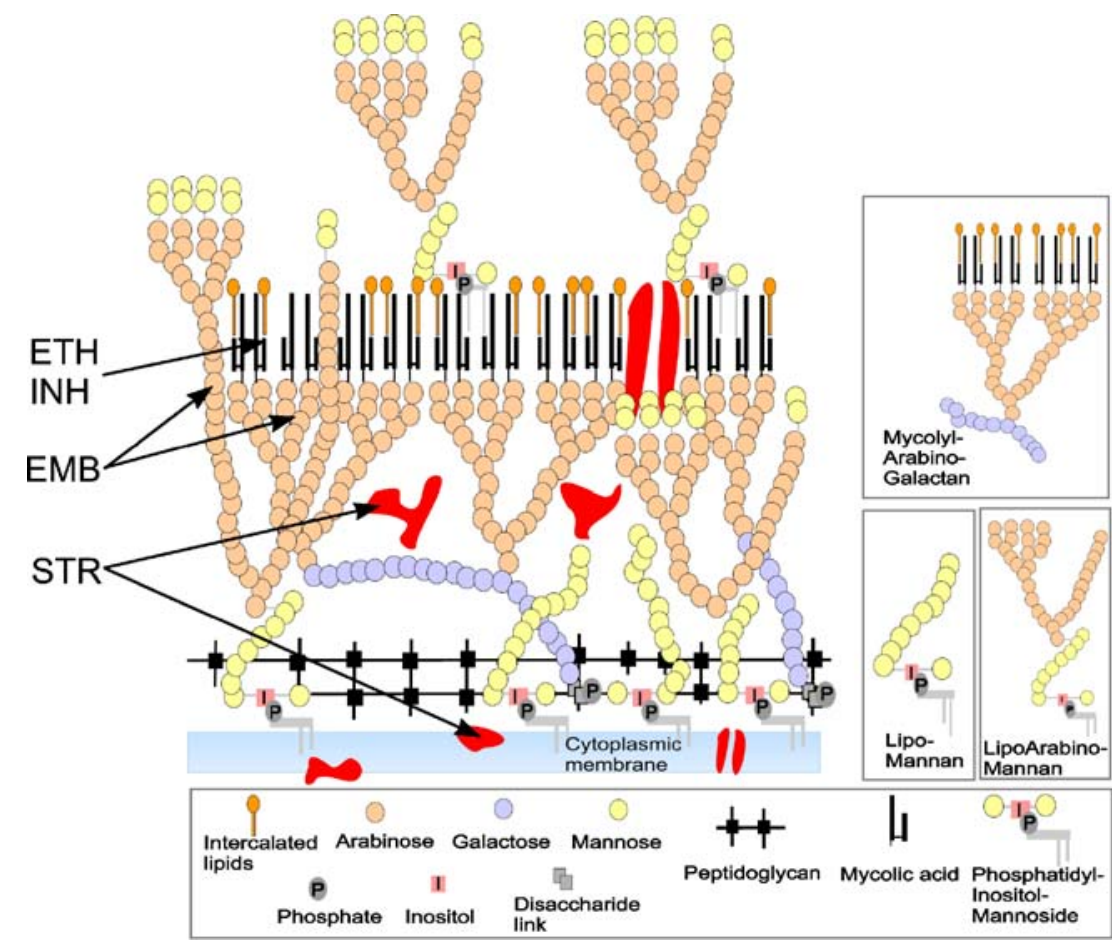

polysaccharides, particularly LAMs, are embedded in this layer $[10,11]$.

In the past few years, atomic force microscopy (AFM) has opened up a range of unprecedented opportunities for imaging and manipulating microbial cells in their native environment [18]. Of particular interest in the pharmacological context is the possibility to directly visualize the effect of drugs on cell surfaces. Early investigations performed in air demonstrated the ability of AFM to visualize drug-induced alterations in the cell walls of Escherichia coli [7], Helicobacter pylori [8], and Staphylococcus aureus [6]. However, attempts to probe cell-drug interaction in situ, i.e., in relevant hydrated conditions, have been very limited so far [28].

The biosynthesis of mycobacterial cell envelope constituents is the site of action of powerful antimycobacterial agents. Ethionamide (ETH) and isoniazid (INH) inhibit the enoyl ACP reductase InhA involved in the biosynthesis of mycolic acids [2], while the synthesis of the glycosylated portion of the wall is efficiently inhibited by ethambutol (EMB), which targets arabinosyltransferases [4]. Streptomycin (STR) targets the 30S subunit of ribosomes, leading to an inhibition of protein synthesis [30].

The aim of the present study was to gain a better understanding of how major antimycobacterial antibiotics alter the mycobacterial cell envelope and, in turn, to refine our molecular view of the mycobacterial wall architecture. To address these issues, three complementary AFM modes were used, i.e., topographic imaging, chemical force microscopy (CFM), and immunogold detection. AFM imaging combined with quantitative roughness analysis revealed that $\mathrm{INH}, \mathrm{ETH}, \mathrm{EMB}$, and STR induce major alterations of the mycobacterial surface. The CFM technique allowed us to correlate these structural changes with variations of chemical properties. Lastly, immunogold detection enabled us to map the distribution of LAM, both on native and treated cells. Our data show that the combination of antibiotic treatments with AFM imaging/ force spectroscopy constitutes a powerful tool to reveal the very complex architecture of the mycobacterial cell wall. Ultimately, these noninvasive nanoscale investigations may help us to understand how structural alterations of the envelope may lead to cell death.

\section{Materials and methods}

\section{Bacterial cultures}

Mycobacterium bovis BCG (strain 1173P2, World Health Organization, Stockholm, Sweden) was grown in Sauton medium. Mycobacteria were cultured at $37^{\circ} \mathrm{C}$ for about 2 weeks in static conditions using $50-\mathrm{cm}^{2}$ Roux flasks that contained $50 \mathrm{~mL}$ of Sauton medium supplemented with Triton WR1339 (Sigma-Aldridch, St. Louis, MO, USA). For some experiments, cells were resuspended for $24 \mathrm{~h}$ in Sauton medium containing INH, ETH, EMB, and STR at concentrations corresponding to minimum inhibitory concentration $(\mathrm{MIC}) / 10, \mathrm{MIC}$, and $10 \times \mathrm{MIC}$. MIC values were $0.02,2,10$, and $15 \mu \mathrm{g} / \mathrm{mL}$ for INH, ETH, EMB, and STR, respectively. All cells were harvested by centrifugation, 
washed three times with milliQ water, and resuspended to a concentration of $\sim 10^{8}$ cells per milliliter.

\section{Atomic force microscopy}

AFM images and force-distance curves were recorded at room temperature $\left(20^{\circ} \mathrm{C}\right)$ in deionized water using a Nanoscope IV Multimode AFM (Veeco Metrology Group, Santa Barbara, CA, USA). We used oxide-sharpened microfabricated $\mathrm{Si}_{3} \mathrm{~N}_{4}$ cantilevers with spring constants of $0.01 \mathrm{~N} / \mathrm{m}$ (Microlevers, Veeco Metrology Group). Mycobacteria were immobilized onto Isopore polycarbonate membranes (Millipore, Billerica, MA, USA) [17, 20]. After filtering a concentrated cell suspension, the filter was gently rinsed with deionized water, carefully cut $(1 \times 1 \mathrm{~cm})$, and attached to a steel sample puck (Veeco Metrology Group) using a small piece of adhesive tape, and the mounted sample was transferred into the AFM liquid cell.

For CFM, cantilevers were coated by electron beam thermal evaporation with a 5-nm-thick Cr layer followed by a 30-nm-thick $\mathrm{Au}$ layer. Gold-coated cantilevers were immersed for $14 \mathrm{~h}$ in $1-\mathrm{mM}$ solutions of $\mathrm{HS}\left(\mathrm{CH}_{2}\right)_{11} \mathrm{CH}_{3}$ in ethanol and then rinsed with ethanol. Adhesion maps and histograms were obtained by recording $16 \times 16$ or $32 \times 32$ force-distance curves on areas of given size and calculating the adhesion force for each force curve.

The procedure for immunogold detection was as follows: Cells immobilized on membranes were preincubated for $1 \mathrm{~h}$ and 30 min with $10 \%$ BSA in phosphate-buffered saline (PBS) to minimize nonspecific adsorption. Samples were then incubated for $2.5 \mathrm{~h}$ with monoclonal mouse anti-LAM antibodies (04.CS.40.1.21.LAM.mm, Colorado State University, USA) diluted in PBS (1:20). Samples were washed three times with PBS and then further incubated for $2.5 \mathrm{~h}$ with the corresponding goat antimouse second antibody conjugated to 10-nm gold particles (Goat-anti-Mouse IgG + IgM, 810.044, Aurion, Wageningen, The Netherlands). After washing three times with PBS, samples were mounted onto steel sample pucks as describe above.

\section{Results and discussion}

Antimycobacterial drugs induce major ultrastructural changes

Using in situ AFM imaging, the ultrastructure of the surface of $M$. bovis $\mathrm{BCG}$ was visualized prior to and after treatment with INH, ETH, EMB, and STR. Mycobacteria were mechanically immobilized onto polycarbonate membranes and imaged in aqueous solution without pretreatment, such as air drying or chemical fixation. Figure 2 shows representative low- and high-resolution images of native $M$.
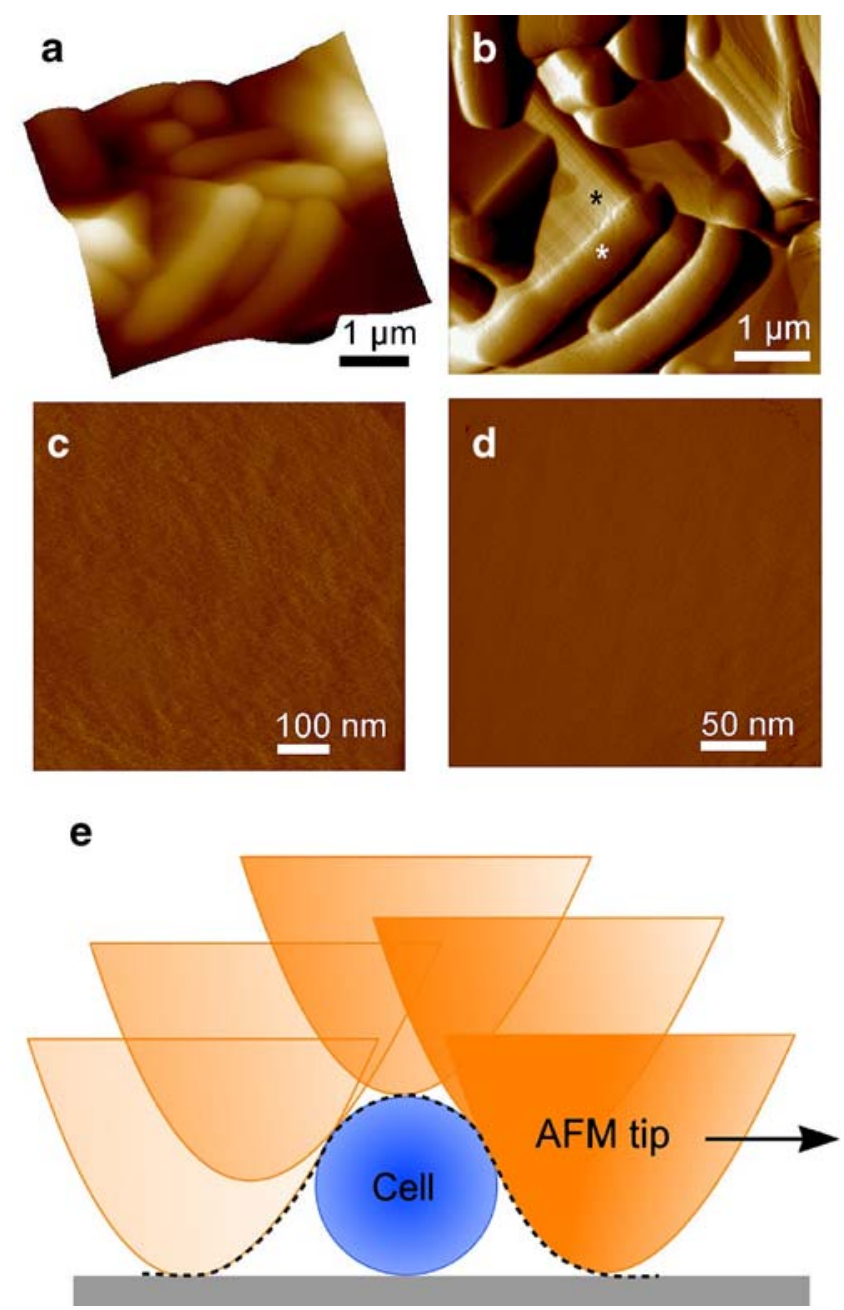

Fig. 2 Topographic imaging of Mycobacterium bovis BCG. a 3-D height image of $M$. bovis BCG cells recorded in contact mode in water. b-d Low- and high-resolution deflection images revealing homogeneous and smooth cell surfaces. The white asterisk in b shows the top part of a cell, while the black asterisk reflects tip convolution artifacts that are schematically described in $\mathbf{e}$

bovis BCG cells. Due to the large curvature of the cells, height-mode images (Fig. 2a) had fairly poor resolution, while deflection-mode images (Fig. 2b-d) were much more sensitive to the surface relief. At low resolution (Fig. 2b), bacteria were surrounded by artifactual structures resulting from the contact between the AFM tip and the edges of the cells (Fig. 2e). High-resolution images revealed a very smooth and homogeneous surface, consistent with previous work [28].

We then imaged mycobacteria following incubation for $24 \mathrm{~h}$ with INH, ETH, EMB, and STR at concentrations corresponding to the MICs (Fig. 3). While the overall integrity of the cells was generally maintained, major ultrastructural alterations were observed: layered structures, striations, and porous morphologies. Presumably, these modifications reflect the inhibition of the synthesis of three major cell wall constituents, i.e., mycolic acids (INH, 

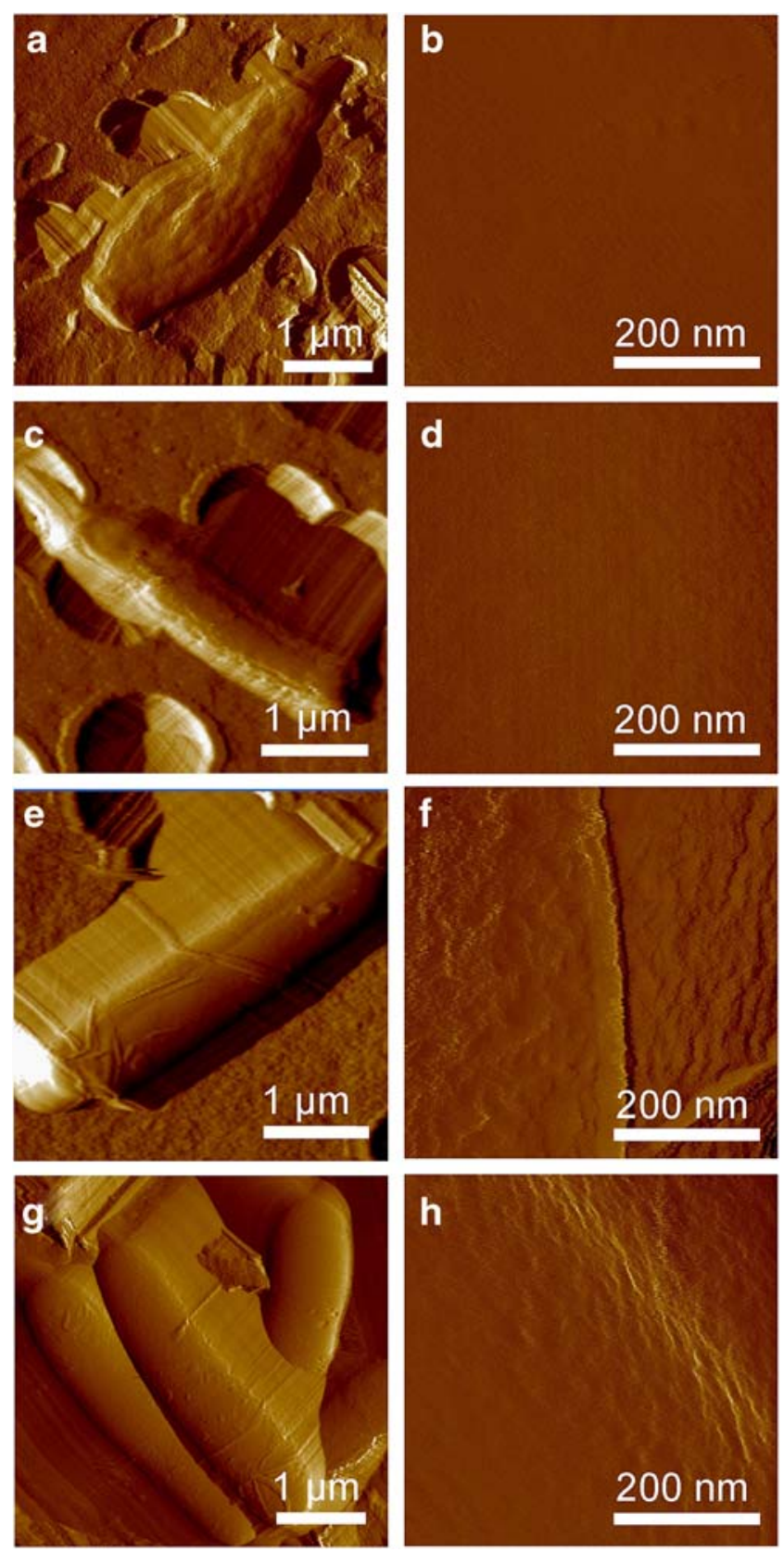

Fig. 3 Topographic imaging of $M$. bovis BCG following treatment with antibiotics. Low- and high-resolution deflection images in aqueous solution of $M$. bovis BCG cells after treatment for $24 \mathrm{~h}$ with $\operatorname{INH}(\mathbf{a}, \mathbf{b}), \operatorname{ETH}(\mathbf{c}, \mathbf{d}), \operatorname{EMB}(\mathbf{e}, \mathbf{f})$, and STR $(\mathbf{g}, \mathbf{h})$ at the MIC. All the drugs induced substantial modifications of the cell surface. For each drug, the images shown are representative of a total of 10 images obtained from at least two independent experiments

ETH), arabinans (EMB), and proteins (STR). It is important to note that comparison of images obtained with the different drugs was qualitative and subjective and that substantial variations were observed when comparing different cells or different preparations.

Therefore, quantitative information from AFM topographic images was extracted by performing statistical analysis of the images at various length scales. To this end, surface roughness was analyzed through the power spectral density (PSD) of the fast Fourier transform of the height images $[14,29]$. Figure 4 shows the variation of rms roughness $\left(R_{\mathrm{rms}}\right)$ as a function of the length scale for native cells and cells treated by the different drugs. For all cells, the surface roughness increased with the length scale and then reached a plateau after $200 \mathrm{~nm}$. Notably, major differences in roughness were observed when comparing the different conditions. While native cells showed subnanometer roughness $\left(R_{\mathrm{rms}}\right.$ of $0.5 \mathrm{~nm}$ on 500 -by-500-nm areas), cells treated with INH and ETH were substantially rougher $\left(R_{\mathrm{rms}}\right.$ of $\left.1 \mathrm{~nm}\right)$. Treatments with EMB and STR lead to even larger roughness values $\left(R_{\mathrm{rms}}\right.$ of 3 and $4 \mathrm{~nm}$, respectively). Interestingly, we note that the increase in surface roughness correlated with the localization of the targets: while moderate changes were observed for INH and ETH, which both target mycolic acids, more pronounced modifications were found for EMB and STR, which target arabinogalactan and proteins.

We also investigated the effect of drug concentration by comparing images obtained at $\mathrm{MIC} / 10, \mathrm{MIC}$, and $10 \times \mathrm{MIC}$ (Fig. 5). Incubation with sub-MIC concentrations of ETH and INH did not significantly alter the surface roughness, while in similar conditions, STR caused a substantial increase of roughness (from 0.5 to $2 \mathrm{~nm}$ ). These results suggest that breakdown of the mycobacterial cell wall can be obtained with antibiotics that do not specifically target the cell wall and that this effect may occur even in conditions where growth is not affected. This observation can be linked to the phenomenon described recently by Possoz et al. [24], showing that sublethal concentrations of bactericidal drugs can deeply affect cellular processes

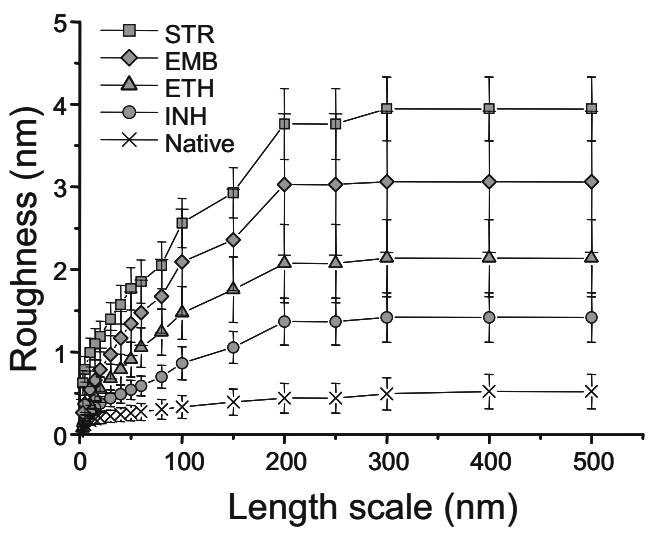

Fig. 4 Statistical analysis of topographic images obtained for $M$. bovis BCG prior to and after drug treatment. Variation of the rms roughness $\left(R_{\mathrm{rms}}\right)$ constructed from power spectral density analysis as a function of the length scale. All drugs give rise to a substantial increase of cell surface roughness, to an extent related to the localization of the target. Each data point represents the mean $\pm \mathrm{SD}$ of three or four images obtained from at least two independent preparations 
Fig. 5 Effect of INH (a), ETH (b), and STR (c) concentrations on the variation of cell surface roughness $\left(R_{\mathrm{rms}}\right)$ constructed from power spectral density analysis at different length scales
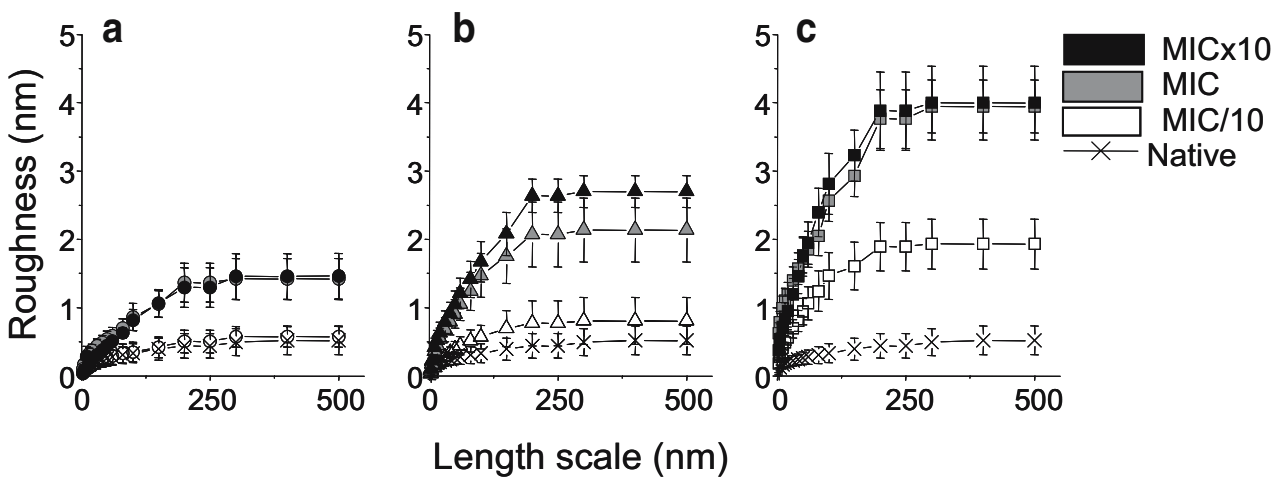

without causing mortality, at least in vitro. The implication of this finding is of importance, as it suggests that antibiotic treatment, even with compounds that are not specifically targeting the cell envelope biosynthesis, may rapidly modify its structural properties and consequently may change the sensitivity of the bacteria to other drugs.

For the three drugs, cells became significantly rougher upon treatment with MIC and $10 \times \mathrm{MIC}$ concentrations. No major differences were observed between the two concentrations, suggesting that increasing the concentration at values larger than the MIC does not change the drug effects. In summary, the above quantitative surface roughness analysis demonstrates that all drugs induced major cell-surface alterations to an extent that correlates with the localization of the target.

Structural changes correlate with differences in chemical properties

We then addressed the question as to whether the drugs alter the cell surface chemical properties, an issue of particular relevance because mycolic acids are thought to confer strong hydrophobic properties to the cells. CFM with hydrophobic tips was used to probe the local hydrophobic character of $M$. bovis BCG prior to and after treatment with INH and EMB for $24 \mathrm{~h}$ at the MIC. As shown in Fig. 6a-c, native cells displayed large adhesion forces $(3,032 \pm 102 \mathrm{pN})$ that were uniformly distributed on the surface. Comparison with data obtained on model alkanethiol surfaces indicates that the bacterial surface is markedly hydrophobic (equivalent to $40 \%$ of hydrophobic methyl groups), which agrees well with the presence of an external layer of mycolic acids. This strong hydrophobicity is not consistent with models describing a bacterial surface exposing polar head groups of associated lipids interacting with mycolic acids [16]. In addition, the adhesion contrast was rather homogeneous, suggesting strongly that native cells are homogeneously hydrophobic and, thus, that mycolic acids form a homogeneous layer with no (or few) hydrophilic polysaccharides [10].
These nanoscale measurements are consistent with earlier macroscopic water contact angle data [5], which showed that the presence of mycolic acids of bacteria from Corynebacterium and Mycobacterium genera is related to cell surface hydrophobicity as well as to cell adhesion to defined surfaces. Mycobacterial species were shown to exhibit water contact angles of 85 to $98^{\circ}$, reflecting remarkably strong hydrophobic properties, while bacteria lacking mycolic acids were much less hydrophobic. Although the water contact angle approach is very useful for assessing the hydrophobic qualities of microorganisms, it provides averaged information obtained on large ensembles of cells. Accordingly, AFM is a powerful complementary approach to such traditional methods, providing for the first time spatially resolved and quantitative measurements of hydrophobicity on single live cells.

Notably, Fig. $6 \mathrm{~d}-\mathrm{i}$ shows that treatment with INH and EMB markedly decreased the hydrophobic character of the cells, adhesion forces of only $137 \pm 31$ and $167 \pm 56 \mathrm{pN}$ being recorded in these conditions. We believe the measured strong hydrophilic properties reflect the exposure of inner-cell-wall carbohydrates, such as arabinose, galactose, and mannose, resulting from the removal of the outermost mycolic acid layer. This observation is consistent with the action modes of the drugs, i.e., inhibition of mycolic acid (INH) and arabinan (EMB) synthesis, as well as with the structural changes observed by topography imaging.

Interestingly, most cells showed homogeneously dark adhesion contrasts (Fig. 6e and h), indicating that the whole mycolic acid layer had been removed or deconstructed upon treatment. However, some cells showed the coexistence of hydrophobic and hydrophilic regions (Fig. 7), attributed to portions of unaltered mycolic acids and of underlying carbohydrates, respectively. This shows that CFM is able to resolve nanoscale chemical heterogeneities on the cell surface. In summary, the above CFM measurements provide direct, unambiguous confirmation that mycolic acids confer strong hydrophobic properties to the cells and that these properties are lost after treatment with antimycobacterial drugs. 
Fig. 6 CFM of native and treated $M$. bovis BCG cells. a, d, $\mathbf{g}$ Topographic images;

$\mathbf{b}, \mathbf{e}, \mathbf{h}$ adhesion force maps; c, $\mathbf{f}, \mathbf{i}$ adhesion force histograms $(n=512)$, and typical force curves recorded on native cells $(\mathbf{a}-\mathbf{c})$ and on cells treated for $24 \mathrm{~h}$ with INH $(\mathbf{d}-\mathbf{f})$ and EMB $(\mathbf{g}-\mathbf{i})$, using hydrophobic tips. Both drugs caused a dramatic decrease of cell surface hydrophobicity, reflecting the removal of the outermost mycolic acid layer
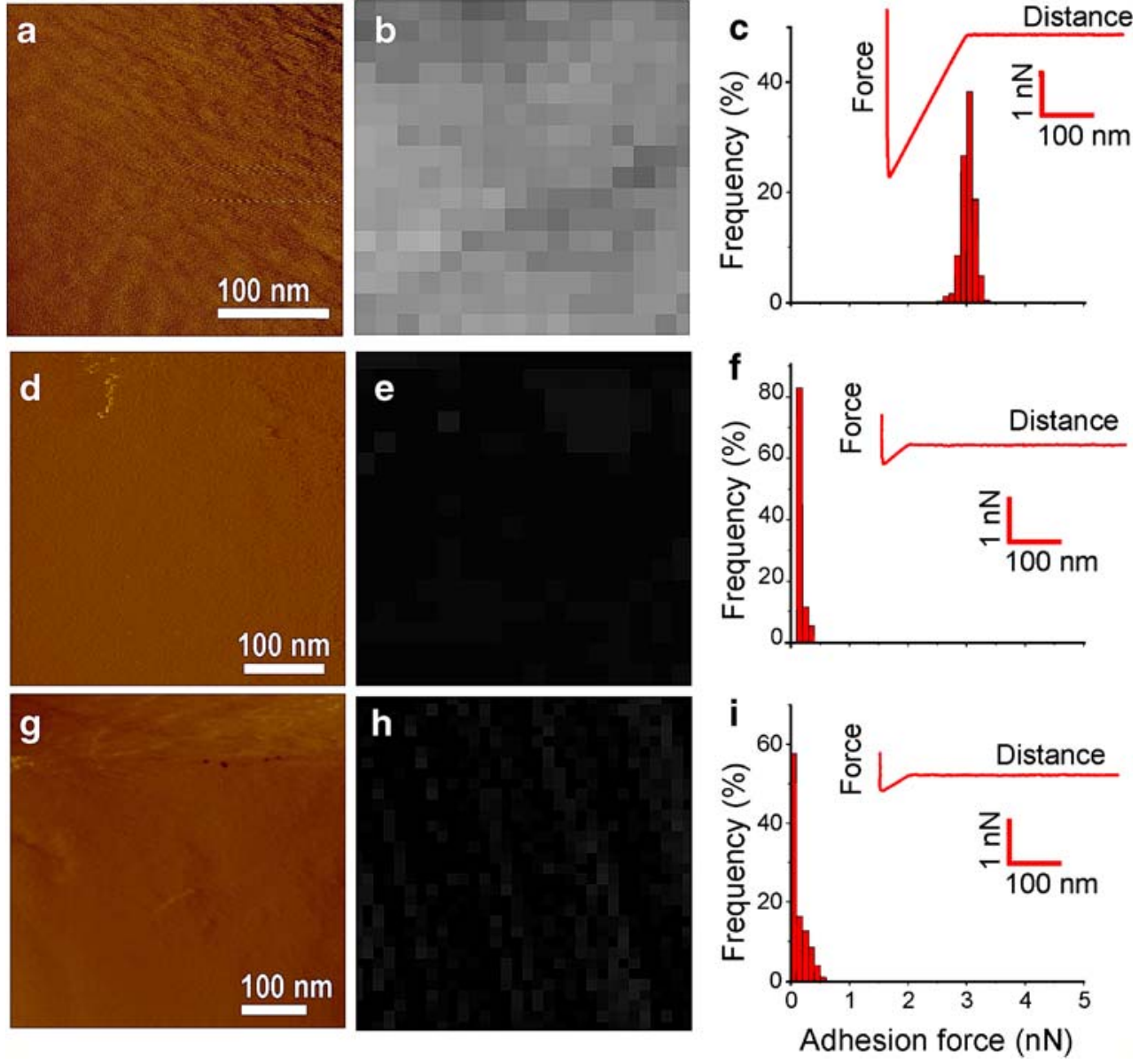

Antimycobacterial drugs lead to the exposure of LAM

Next, we used immunogold AFM imaging to detect and localize LAM on the surface of $M$. bovis BCG, prior to and

after treatment with INH and EMB. To this end, cells were first incubated with monoclonal anti-LAM antibodies and then further incubated with the corresponding gold-conjugated secondary antibodies. To minimize detachment of the loosely
Fig. 7 Resolving nanoscale chemical heterogeneities on treated M. bovis BCG cells. High-resolution images, adhesion force maps, and histograms obtained on cells treated for $24 \mathrm{~h}$ with INH $(\mathbf{a}-\mathbf{c})$ and EMB (d-f), using hydrophobic tips. Cell surfaces showed the coexistence of hydrophobic and hydrophilic regions, attributed to regions of unaltered mycolic acids and of newly exposed carbohydrates, respectively
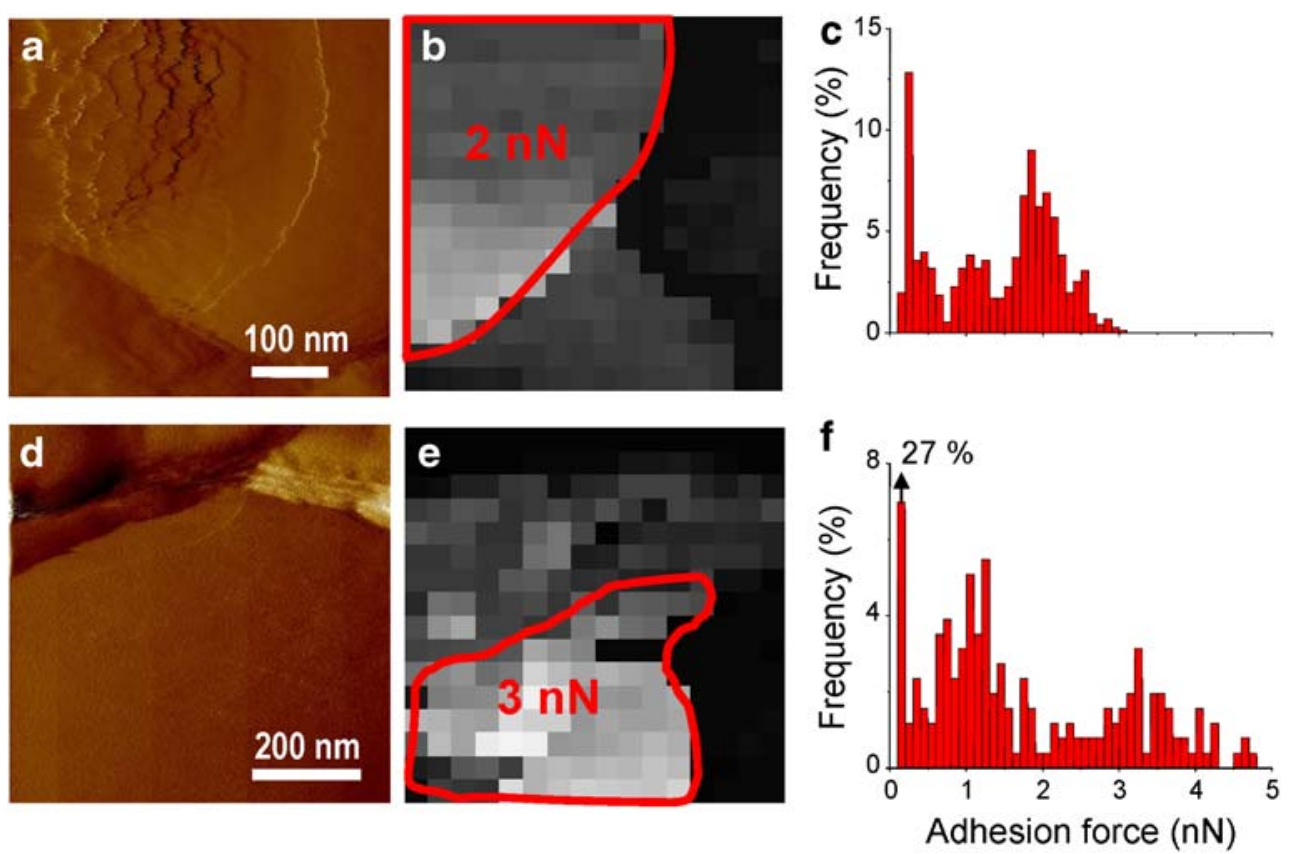
bound particles, we compared images obtained in contact and tapping (phase) modes. Figure 8 presents contact mode and tapping mode images obtained for immunogold-labeled M. bovis BCG cells prior to and after treatment with INH and EMB for $24 \mathrm{~h}$ at the MIC. As can be seen, the surface of native cells showed essentially no labeling, a few isolated gold particles being observed in tapping mode. This finding suggests that LAM is not (or only weakly) exposed on native cell surfaces. By contrast, tapping mode images recorded for INH- and EMB-treated cells revealed a large coverage of gold particles, indicating that LAM was exposed. This observation, which correlates with the above topography and chemical data, demonstrates that INH and EMB treatments lead to the exposure of hydrophilic LAM at the cell surface. For INH, this effect is fully consistent with the action mode of the drug, expected to eliminate the outer mycolic acid layer [2]. For EMB, this finding is more
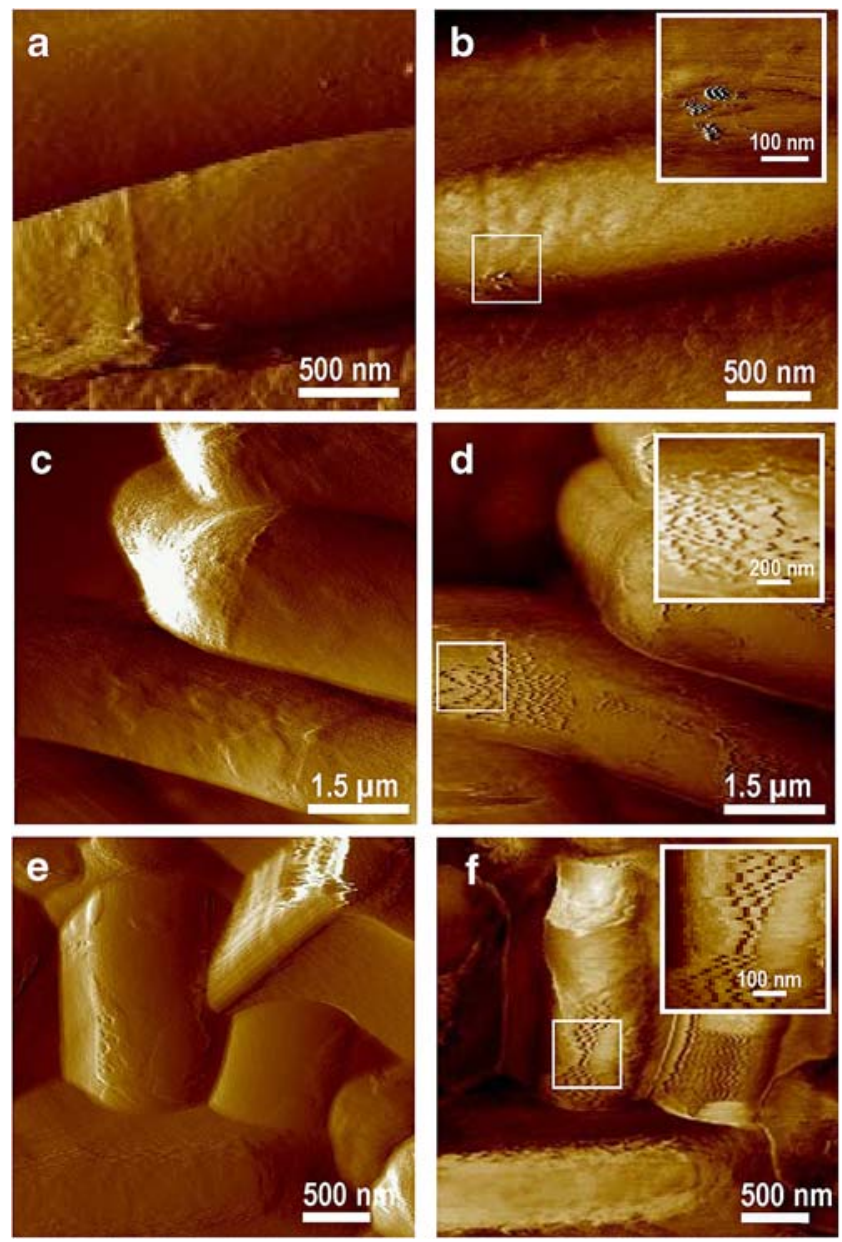

Fig. 8 Detection of lipoarabinomannan (LAM) on native and treated M. bovis BCG cells using immunogold AFM. Contact mode (deflection) images (a, c, e) and tapping mode (phase) images (b, d, f) of immunogold-labeled cells: native cells $(\mathbf{a}, \mathbf{b})$ and cells treated for $24 \mathrm{~h}$ with INH (c, d) and EMB (e, f). All cells were incubated with monoclonal anti-LAM antibodies, followed by another incubation with the corresponding gold-conjugated secondary antibodies surprising because the drug inhibits the polymerization of arabinose found in arabinogalactan and LAM [4]. Because we showed that the drug removes mycolic acids, our data suggest that LAM and arabinogalactan - onto which mycolic acids are attached - are not repressed in the same way, which is actually consistent with previous studies [11, $13,21,22,27]$. In agreement with hydrophobic heterogeneities observed on treated cells (Fig. 7), we noted that some regions were devoid of any particles. Thus, we believe these nonlabeled areas correspond to hydrophobic regions of nonaltered mycolic acids, reflecting the tendency of these molecules to self-organize. Finally, it is also worth noting that: (1) gold particles were never seen in contact mode, emphasizing the need to use tapping (phase) mode for such in situ immunogold studies, and (2) gold particles were poorly resolved and rather fuzzy in tapping mode, presumably reflecting energy dissipation of the oscillating tip.

The feasibility of using immunogold labels as cellsurface markers in AFM studies has been demonstrated earlier. In a pioneering study [25], AFM was used to image the surface of immunogold-labeled human lymphocytes. AFM images revealed colloidal gold particles on the cell surface with and without silver enhancement. Individual immunogold particles were clearly resolved from the cell surface, thus determining the location of antigens. More recently, a similar AFM-based immunogold technique allowed the revealing of types I and II collagen fibers on rat fibroblasts and human chondrosarcoma cells [1]. Because the above cellular studies were performed in the dried state, it is unclear whether the information provided is more relevant than that obtained using conventional immunogold electron microscopy. To our knowledge, the present study represents the first attempt to apply immunogold AFM to hydrated bacterial cells.

Summary and biological implications

AFM - used in the topography, CFM, and immunogold modes - is a powerful multifunctional tool for exploring the interactions between live mycobacteria and antimycobacterial drugs. Quantitative topographic imaging shows that INH, ETH, EMB, and STR induce a major increase of cell surface roughness. Using CFM, these structural alterations are found to correlate with a dramatic decrease of cell surface hydrophobicity, an effect that we attribute, at least for INH and ETH treatments, to the removal of the mycolic acid layer. Consistent with these findings, immunogold detection shows that treatment with these two drugs leads to the massive exposure of hydrophilic LAM at the surface.

Taken together, the above structural, chemical, and immunological data provide novel insight into the 3-D organization of the mycobacterial cell wall. The uniform distribution of cell surface hydrophobicity measured on 
native cells and the absence of any substantial labeling with anti-LAM antibodies strongly suggest that LAM is not abundant at the cell surface, the latter being essentially composed of highly hydrophobic molecules such as mycolic acids or wall-associated lipids. This finding makes it very unlikely that LAM is anchored in the mycolic acid leaflet via its lipid tail and exposed at the surface of the bacteria. This may seem surprising because ELISA tests have shown the presence of LAM at the cell surface [11]. However, this apparent discrepancy may easily be explained as follows: (1) unlike AFM, ELISA assays are not specific to the outermost cell surface but probe the entire cell wall, and (2) as opposed to here, ELISA measurements may have been performed in conditions favoring a nonspecific anchoring of LAM to the cell wall. The question as to how these glycolipids, as well as other components of the external portion of the envelope, cross the outer lipid barrier remains to be addressed.

To our knowledge, this is the first time that modifications of the physicochemical properties of the cell wall due to antibiotic treatments were observed on hydrated mycobacteria. The data confirm the idea that the dual property of the cell wall composed of an outer homogenously hydrophobic leaflet of mycolic acid and an inner hydrophilic arabinogalactan matrix should eventually limit the passage of both hydrophobic and hydrophilic antibiotics. Consequently, the development of new antimycobacterial compounds should take into account their future association with drugs that modify the ultrastructure of the cell wall and that may influence their capacity to cross the remaining envelope. On the other hand, this work also suggests that the efficiency of a given therapy will progressively change with the erosion of the envelope, and not necessarily in a positive manner.

Acknowledgements This work was supported by the National Foundation for Scientific Research (FNRS); the Université catholique de Louvain (Fonds Spéciaux de Recherche), the Région wallonne; the Federal Office for Scientific, Technical and Cultural Affairs (Interuniversity Poles of Attraction Programme); Institut National de la Santé et de la Recherche Médicale; and the Research Department of the Communauté française de Belgique (Concerted Research Action). Y.F. D. and D.A. are Research Associate and Research Fellow of the FNRS, respectively. Anti-LAM antibodies were kindly provided by the Colorado State University as part of NIH, National Institute of Allergy and Infectious Diseases contract no. HHSN266200400091C, entitled "Tuberculosis Vaccine Testing and Research Materials."

\section{References}

1. Arntz Y, Jourdainne L, Greiner-Wacker G, Rinckenbach S, Ogier J, Voegel JC, Lavalle P, Vautier D (2006) Immunogold detection of types I and II chondrocyte collagen fibrils: an in situ atomic force microscopic investigation. Microsc Res Tech 69:283-290
2. Banerjee A, Dubnau E, Quemard A, Balasubramanian V, Um KS, Wilson T, Collins D, Delisle G, Jacobs WR (1994) InhA, a gene encoding a target for isoniazid and ethionamide in Mycobacterium tuberculosis. Science 263:227-230

3. Baulard AR, Besra GS, Brennan PJ (1999) The cell wall core of Mycobacterium: structure, biogenesis, and genetics. In: Ratledge C, Dale J (eds) Mycobacteria: molecular biology and virulence. Blackwell, Oxford, pp 240-259

4. Belanger AE, Besra GS, Ford ME, Mikusova K, Belisle JT, Brennan PJ, Inamine JM (1996) The embAB genes of Mycobacterium avium encode an arabinosyl transferase involved in cell wall arabinan biosynthesis that is the target for the antimycobacterial drug ethambutol. Proc Natl Acad Sci USA 93:11919-11924

5. Bendinger B, Rijnaarts HHM, Altendorf K, Zehnder AJB (1993) Physicochemical cell-surface and adhesive properties of Coryneform bacteria related to the presence and chain-length of mycolic acids. Appl Environ Microbiol 59:3973-3977

6. Boyle-Vavra S, Hahm J, Sibener SJ, Daum RS (2000) Structural and topological differences between a glycopeptide-intermediate clinical strain and glycopeptide-susceptible strains of Staphylococcus aureus revealed by atomic force microscopy. Antimicrob Agents Chemother 44:3456-3460

7. Braga PC, Ricci D (1998) Atomic force microscopy: application to investigation of Escherichia coli morphology before and after exposure to cefodizime. Antimicrob Agents Chemother 42:18-22

8. Braga PC, Ricci D (2000) Detection of rokitamycin-iduced morphostructural alterations in Helicobacter pylori by atomic force microscopy. Chemotherapy 46:15-22

9. Brennan PJ (2003) Structure, function, and biogenesis of the cell wall of Mycobacterium tuberculosis. Tuberculosis 83:91-97

10. Brennan PJ, Crick DC (2007) The cell-wall core of Mycobacterium tuberculosis in the context of drug discovery. Curr Top Med Chem $7: 475-488$

11. Chatterjee D (1997) The mycobacterial cell wall: structure, biosynthesis and sites of drug action. Curr Opin Chem Biol 1:579-588

12. Daffe M, Draper P (1998) The envelope layers of mycobacteria with reference to their pathogenicity. Adv Microb Physiol 39:131-203

13. Deng LY, Mikusova K, Robuck KG, Scherman M, Brennan PJ, McNeil MR (1995) Recognition of multiple effects of ethambutol on metabolism of mycobacterial cell envelope. Antimicrob Agents Chemother 39:694-701

14. Denis FA, Hanarp P, Sutherland DS, Gold J, Mustin C, Rouxhet PG, Dufrêne YF (2002) Protein adsorption on model surfaces with controlled nanotopography and chemistry. Langmuir 18:819-828

15. Dinadayala P, Kaur D, Berg S, Amin AG, Vissa VD, Chatterjee D, Brennan PJ, Crick DC (2006) Genetic basis for the synthesis of the immunomodulatory mannose caps of lipoarabinomannan in Mycobacterium tuberculosis. J Biol Chem 281:20027-20035

16. Draper P (1998) The outer parts of the mycobacterial envelope as permeability barriers. Front Biosci 3:1253-1261

17. Dufrêne YF (2002) Atomic force microscopy, a powerful tool in microbiology. J Bacteriol 184:5205-5213

18. Dufrêne YF (2004) Using nanotechniques to explore microbial surfaces. Nat Rev Microbiol 2:451-460

19. Dye C (2006) Global epidemiology of tuberculosis. Lancet 367:938-940

20. Kasas S, Ikai A (1995) A method for anchoring round shaped cells for atomic-force microscope imaging. Biophys J 68:1678-1680

21. Khoo KH, Douglas E, Azadi P, Inamine JM, Besra GS, Mikusova K, Brennan PJ, Chatterjee D (1996) Truncated structural variants of lipoarabinomannan in ethambutol drug-resistant strains of Mycobacterium smegmatis-inhibition of arabinan biosynthesis by ethambutol. J Biol Chem 271:28682-28690

22. Mikusova K, Slayden RA, Besra GS, Brennan PJ (1995) Biogenesis of the mycobacterial cell wall and the site of action of ethambutol. Antimicrob Agents Chemother 39:2484-2489 
23. OrtaloMagne A, Lemassu A, Laneelle MA, Bardou F, Silve G, Gounon P, Marchal G, Daffe M (1996) Identification of the surface-exposed lipids on the cell envelopes of Mycobacterium tuberculosis and other mycobacterial species. J Bacteriol 178:456-461

24. Possoz C, Newmark J, Sorto N, Sherratt DJ, Tolmasky ME (2007) Sublethal concentrations of the aminoglycoside amikacin interfere with cell division without affecting chromosome dynamics. Antimicrob Agents Chemother 51:252-256

25. Putman CAJ, Degrooth BG, Hansma PK, Vanhulst NF, Greve J (1993) Immunogold labels: cell-surface markers in atomic force microscopy. Ultramicroscopy 48:177-182

26. Sieling PA, Chatterjee D, Porcelli SA, Prigozy TI, Mazzaccaro RJ, Soriano T, Bloom BR, Brenner MB, Kronenberg M, Brennan PJ,
Modlin RL (1995) CD1-Restricted T-cell recognition of microbial lipoglycan antigens. Science 269:227-230

27. Strohmeier GR, Fenton MJ (1999) Roles of lipoarabinomannan in the pathogenesis of tuberculosis. Microbes Infect 1:709-717

28. Verbelen C, Dupres V, Menozzi FD, Raze D, Baulard AR, Hols P, Dufrêne YF (2006) Ethambutol-induced alterations in Mycobacterium bovis BCG imaged by atomic force microscopy. FEMS Microbiol Lett 264:192-197

29. Viville P, Lazzaroni R, Bredas JL, Moretti P, Samori P, Biscarini F (1998) The influence of thermal annealing on the morphology of sexithienyl thin films. Adv Mater 10:57-60

30. Zhang Y, Post-Martens K, Denkin S (2006) New drug candidates and therapeutic targets for tuberculosis therapy. Drug Discov Today 11:21-27 